\title{
Bilateral Nephrocalcinosis in Primary Distal Renal Tubular Acidosis
}

\author{
Siwadon Pitukweerakul, MD ${ }^{7}$ and Sittichoke Prachuapthunyachart, MD² \\ 'Department of Medicine, Presence Saint Francis Hospital, Evanston, IL, USA; ${ }^{2}$ Department of Pediatrics, University of South Alabama, Mobile, AL, \\ USA.
}

KEY WORDS: nephrocalcinosis; distal renal tubular acidosis; clinical image.

J Gen Intern Med 31(10): 1261

DOI: $10.1007 /$ s11606-016-3697-z

(C) Society of General Internal Medicine 2016

\section{CLINICAL DESCRIPTION}

A 48-year-old White man with a history of congenital distal renal tubular acidosis and diabetes presented with low urine output for 6 months. He was diagnosed with distal renal tubular acidosis when he was 9 years old. He was not compliant to his alkali treatment. Labs were notable for a creatinine of $7 \mathrm{mg} / \mathrm{dL}$, serum bicarbonate of $13 \mathrm{mEq} / \mathrm{L}$ with a positive urine anion gap and urine $\mathrm{pH}$ of 6 . Plain radiographs of the abdomen and renal ultrasonograph are shown in Figs. 1 and 2, respectively.

Nephrocalcinosis is a rare condition in which there is calcium deposited in the kidneys. It can be seen in a variety of congenital and acquired diseases. ${ }^{1}$ Diagnosis is often made incidentally by imaging such as plain abdominal radiography, kidney ultra-

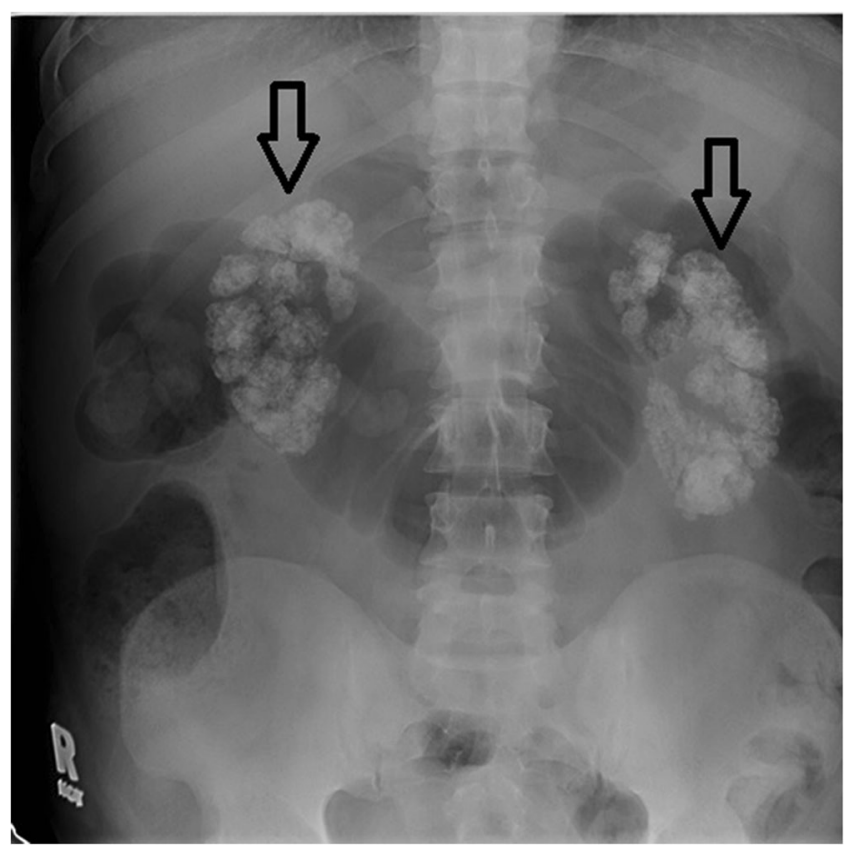

Figure 1. Plain radiography of the abdomen demonstrating bilateral nephrocalcinosis (transparent arrow).

Received February 22, 2016

Revised March 17, 2016

Accepted March 29, 2016

Published online April 18, 2016

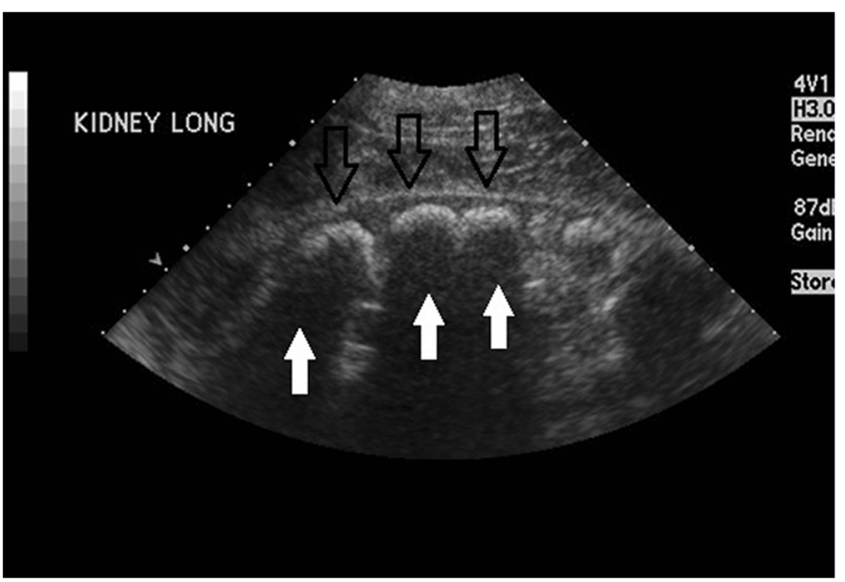

Figure 2. Renal ultrasonography in the longitudinal plane demonstrating calcification in the renal pyramids (transparent arrow) with acoustic shadowing (white arrow).

sonography and abdominal computed tomography. ${ }^{2}$ In general, nephrocalcinosis is recognized as systemic disease and appropriate evaluation is needed to clarify underlying disease. ${ }^{2}$ Differential diagnosis of nephrocalcinosis includes primary hyperparathyroidism, sarcoidosis, hypervitaminosis D, medullary sponge kidney, distal renal tubular acidosis and other inherited tubulopathies. Initial laboratory evaluation including urinalysis, urine electrolyte, urine anion gap, serum calcium and phosphate, parathyroid hormone level and serum vitamin D level should be obtained based on clinical suspicion. ${ }^{1}$

Contributors: We have no additional contributors other than those listed as Authors.

Corresponding Author: Siwadon Pitukweerakul, MD; Department of MedicinePresence Saint Francis Hospital, 355 Ridge avenue, Evanston, IL 60202, USA (e-mail: siwadon.pituk@gmail.com). Compliance with Ethical Standards:

Funders: We have no funding sources to declare.

Prior Presentations: This Clinical Image has never been presented at a conference or accepted for publication by another journal or institution.

Conflict of Interest: The authors declare that they do not have a conflict of interest.

\section{REFERENCES}

1. Shavit L, Jaeger P, Unwin RJ. What is nephrocalcinosis? Kidney Int. 2015;88(1):35-43.

2. Hsi RS, Stoller ML. A spectrum: nephrocalcinosis-nephrolithiasis. J Urol. 2015;194(5):1188-9. 